PO 8447 ROBUST CLINICAL TRIALS ARE NOT ENOUGH: OVERCOMING OPERATIONAL CHALLENGES FOR IMPLEMENTING REMSTART INTERVENTION PACKAGE (TRIP STUDY) INTO ROUTINE PRACTICE

${ }^{1}$ Sokoine Kivuyo*, ${ }^{1}$ Frank Erick, ${ }^{2}$ Angela Loyse, ${ }^{3}$ Shabar Jaffar, ${ }^{1}$ Godfrey S Mfinanga ${ }^{1}$ National Institute for Medical Research-Muhimbili Centre, Dar es Salaam, Tanzania; ${ }^{2}$ St George's University of London, UK; ${ }^{3}$ Liverpool School of Tropical Medicine, Liverpool, UK

10.1136/bmjgh-2019-EDC.101

Background The REMSTART trial identified an effective package (cryptococcal antigen (CrAg) screening and enhanced antiretroviral therapy (ART) adherence support) that reduced allcause mortality in advanced HIV (CD4 $\leq 200$ cells $/ \mathrm{mm}^{3}$ ) by $28 \%$ compared to standard of care. The introduction of this package at clinic level has been necessary to impact routine care practices in Tanzania.

Methods The TRIP study is cluster-randomised. The intervention package was implemented in 16 routine care facilities (early arm) whilst 8 facilities continued with standard of care (deferred arm). At the end of 12 months follow-up, the intervention was implemented in the deferred facilities. The primary endpoint is all-cause mortality at 1 year.

Results Implementation of the REMSTART intervention into routine care services has highlighted the following challenges: 1) Baseline CD4 testing: half (4/8) of rural facilities had no CD4 machines and in a further $3 / 8$ there was a lack of reagents needed for CD4 testing. Clinical staging has replaced inclusion criterion where CD4 testing is not available; 2) Heavy staff workload in routine care; regular discussion with policymakers and workshops enhanced the take-up of the package; 3) Timing of ART: the Ministry of Health has updated national guidelines to include the package and delay ART by 2 weeks in CrAg-positives.

Conclusion It has proven essential to engage with policymakers and programme managers from the outset, i.e. during the REMSTART trial itself and the following TRIP implementation study. The Ministry of Health has now changed the national HIV guidelines to include the REMSTART package and develop training modules for $\mathrm{CrAg}$ screening in all regional hospitals. The TRIP study has revealed key issues that must be addressed to allow scaling up the interventions.

\section{PO 8448 ADVERSE DRUG REACTION TO TWO ARTEMISININ- BASED COMBINATION THERAPIES, ARTEMETHER- LUMEFANTRINE AND ARTEMISININ-PIPERAQUINE, IN CHILDREN WITH ACUTE UNCOMPLICATED MALARIA IN IBADAN, NIGERIA}

Oluwafunmibi Anjorin*, Catherine 0 Falade. Department of Pharmacology and Therapeutics, University of Ibadan, Ibadan, Nigeria

10.1136/bmjgh-2019-EDC.102

Background Malaria remains a public health problem in subSaharan Africa, especially amongst children and pregnant women. Artemisinin-based combination therapy (ACT) is now the treatment of choice. Adverse drug reactions (ADR) have been observed to ACTs. This study aims to determine the incidence, pattern of presentation and factors associated with ADRs to artemether-lumefantrine (AL) and artemisinin-piperaquine (AP) among children with acute uncomplicated malaria in Ibadan, Nigeria.
Methods Children aged 2-10 years with acute uncomplicated malaria who met the inclusion criteria, were enrolled, randomised to receive one of the study drugs (AL or AP) and followed up for 28 days. Monitoring for ADR was based on history from the parent/guardian and/or child regarding occurrences of treatment emergent signs and symptoms and on abnormalities of laboratory investigations (full blood count and blood chemistry). Causality assessment for the ADR was by the Naranjo algorithm scale while the severity was assessed using the Hartwig's severity scale.

Results A total of 114 children were enrolled; six defaulted and were not available for follow-up. There were 61 (56.5\%) males. The mean age of enrollees was $65.1 \pm 30.0$ months. Fever was the most prevalent presenting complaint occurring in $108(100 \%)$ enrollees. Observed ADRs were cough, diarrhoea, loss of appetite, abdominal pain, rash, fever, irritability, insomnia and headache but the differences were not statistically significant between the two groups. The incidence of ADR to both ACTs was $12 / 1000$ patients per day. Prevalence of ADR to AL was $14 \%$ and for AP $11 \%$; this was not statistically significant. All ADRs were mild. No notable associated factor to ADR was detected in this study.

Conclusion Both AL and AP were found to be safe in the study population.

\section{PO 8449 SCHISTOSOMA HAEMATOBIUM INFECTION INCREASES THE NUMBER OF MALARIA EPISODES IN CHILDREN LIVING IN RURAL AREAS AROUND LAMBARÉNÉ, GABON}

${ }^{1}$ Jean Claude Dejon Agobé*, ${ }^{1}$ Jean R Edoa, ${ }^{1}$ Yabo J Honkpehedji, ${ }^{1}$ Jeannot Fréjus Zinsou, 'Bayodé R Adegbité, 'Bertrand Lell, ${ }^{2}$ Martin P Grobusch, ${ }^{3}$ Benjamin Mordmüller, ${ }^{1}$ Ayola Akim Adegnika. 'Centre de Recherches Médicales de Lambaréné (CERMEL), Libreville, Gabon; 'Academic Medical Center, University of Amsterdam, the Netherlands; ${ }^{3}$ University of Tübingen, Germany

\subsection{6/bmjgh-2019-EDC.103}

Background In sub-Saharan Africa, Plasmodium spp. infection prevalence very often overlaps with helminth infections, particularly with schistosomiasis which is reported to be the second parasitic infection after malaria in terms of prevalence. Interaction between both infections has been reported earlier. Schistosomiasis is typically a chronic disease, whereas malaria occurs in episodes, particularly in children. In this study, we assessed the effect of Schistosoma haematobium infection on clinical malaria among children.

Methods A longitudinal study was conducted from June 2016 to February 2018. Volunteers without any known chronic condition were included. Thick blood smear (TBS) was performed monthly at participants' homes. For any medical concern including malaria-like symptoms and visible haematuria, participants were invited to come to CERMEL for diagnosis and treatment. Light microscopy was performed to detect malaria parasites and Schistosoma eggs, using TBS and urine filtration technique, respectively. Over the study course, participants found to be infected were treated accordingly. Schistosomiasis status was determined at the end of the follow-up.

Results Among the 351 volunteers included in the study, schistosomiasis status was available for 260. Mean age was 12.3 year (SD 4.6) with a 0.96 women-to-men sex ratio. Of those, $112 / 260(43.1 \%) \quad[37.0 \%-49.3 \%]$ participants were 
positive for S. haematobium, and a total of 132 (51\%) children developed 230 malaria attacks. Those with schistosomiasis had a $1.5: 1$ [1.1-2.0] risk to develop malaria compared to their uninfected counterparts. The mean number of malaria episodes per child over the study course was higher among children with schistosomiasis compared to those without (2.03 vs 1.57 , p-value $=0.015$ ).

Conclusion S. haematobium infection was associated with increased susceptibility to develop malaria (by increasing the risk to develop a malaria episode) and, consequently, a higher malaria incidence.

\section{PO 8458 POLYMORPHISM OF THE PLASMODIUM FALCIPARUM MSP-2 GENE ASSOCIATED WITH PLACENTAL MALARIA AT THE BORGOU-ALIBORI DEPARTMENTAL HOSPITAL}

Dossou Akpéyédjé*, Saadou Issifou. Fondation pour le Recherche Scientifique, Cotonou, Benin

\subsection{6/bmjgh-2019-EDC.104}

Background In sub-Saharan Africa, malaria during pregnancy is a major health problem because it poses significant risks for the pregnant woman and the foetus. The sequestration of Plasmodium falciparum-infected erythrocytes in the placenta has consequences for the mother and the foetus. This study aimed to evaluate the allelic polymorphism of the Plasmodium falciparum MSP-2 gene related to the consequences of placental malaria.

Methods It was a cross-sectional study conducted over two periods lasting six months in 2016 and 2017. The maternity center of the Hospital of Borgou-Alibori in Benin served as a framework for the study. From the 98 parturients included, placental blood samples were taken and then genotyped.

Results Using the MSP-2 gene as marker, the prevalence was 17, 34\%. The MSP-2 gene was polymorphic with 9 distinct allelic types for both 3D7 and FC27 families (150 bp; 200 bp; 250 bp; 275 bp; 300 bp; 350 bp; 400 bp; 450 bp and $500 \mathrm{bp}$ ). The FC27 allelic family was predominant over the $3 \mathrm{D} 7$ family with $56,25 \%$ and $43,75 \%$ respectively. The 300 bp allelic type (50\%) was predominant in the FC27 family while the $400 \mathrm{bp}$ type was predominant in 3D7 family $(35,71 \%)$. 9 women had polyclonality $(52,94 \%)$. The multiplicity of infection (MOI) was 1, 88. The number of strains ranged 1 to 4 in infected women. In univariate analysis there was no significant relationship between MSP-2 gene polymorphism and maternofoetal consequences. The absence of prenatal consultation $(p=0.0270)$, non-taking of IPTp/SP $(p=0.0060)$, the occurrence of malaria in the third trimester $(p=0,0364)$ and moderate maternal anaemia $(p=0.0277)$ were associated with the polymorphism of MSP-2 gene. The MOI was significantly associated with parasite density of infected women.

Conclusion Plasmodium falciparum MSP-2 gene was polymorphic in infected women at Parakou. Several factors related to pregnancy monitoring were associated with this genetic diversity. It is therefore essential to ensure correct follow-up of pregnancies.

\section{PO 8460 PANDORA-ID NET (PAN-AFRICAN NETWORK FOR RAPID RESEARCH, RESPONSE, RELIEF AND PREPAREDNESS FOR INFECTIOUS DISEASES EPIDEMICS)}

${ }^{1}$ Francine Ntoumi*, ${ }^{2}$ Francine Zumla, ${ }^{3}$ Giuseppe Ippolito, ${ }^{3}$ Francesco Vairo. ${ }^{1}$ Fondation Congolaise pour la Recherche Médicale, Brazzaville, Republic of the Congo; ${ }^{2}$ University College of London, London, UK; ${ }^{3}$ National Institute for Infectious Diseases Lazzaro Spallanzani, Rome, Italy

\subsection{6/bmjgh-2019-EDC.105}

Background New and re-emerging infectious disease outbreaks continue to cause much human suffering and loss of life worldwide. Since Africa has experienced repeated outbreaks of zoonotic infections, an important need exists to improve local and regional capacities to identify and respond to zoonotic outbreaks. PANDORA ID-NET is an EDCTP-supported 'ONE Human and Animal HEALTH' multidisciplinary consortium of 24 partner institutions (15 African and 9 European) in 9 African and 4 European countries.

Methods Our overall aim is to strengthen regional and panAfrican capacities and systems for enabling a rapid and effective response to infectious diseases with epidemic potential, arising from within Africa or imported from overseas. We aim to build laboratory and public health capabilites for rapid detection and surveillance of pathogens from human and animal sources. This will include obtaining accelerated evidence for optimal clinical management of patients, infection control measures, and public health response during outbreaks. Capacities will be built: a) for performing multisite clinical trials (evaluating rapid diagnostics, biomarkers, a range of treatments, vaccines and operational research studies) and, b) for timely collection, analysis and communication of information.

Conclusion Our activities will be aligned to EDCTP regional Networks of Excellence, Africa CDC and other relevant global and regional initiatives, thus maximizing complementarity and achieving a multiplier effect, facilitating rapid policy implementation of outputs.

\section{PO 8467 IMPACT OF COMMUNITY CASE MANAGEMENT OF MALARIA (CCMM) AMONG CHILDREN UNDER FIVE YEARS OLD: AN EVALUATIVE STUDY IN KABONGA}

${ }^{1}$ Ciza Bonne*, ${ }^{2}$ Kagozi Husseni, ${ }^{3}$ Ciza Josephine Muhigirwa. ${ }^{1}$ Health Healing Network Burundi, Bujumbura, Burundi; ${ }^{2}$ Solidarity of nurses for promotion of maternal and children's health, Burundi; ${ }^{3}$ Palliative care association of Burundi, Bujumbura, Burundi

\subsection{6/bmjgh-2019-EDC.106}

Background In Burundi, malaria is considered a major public health concern and the leading cause of death. Malaria is responsible for up to $25 \%$ of all outpatient visits and up to $48 \%$ of all deaths in health facilities among children under five years old. Despite efforts made, timely access to health care is still limited, mainly due to geographic inaccessibility and lack of awareness about malaria complications. To increase timely access to malaria treatment, Burundi's Ministry of Health implemented Community Case Management of Malaria (CCMM) since 2015 in Kabonga. We sought to assess the impact of CCMM in improving malaria outcomes following two years of its initiation. 